See discussions, stats, and author profiles for this publication at: https://www.researchgate.net/publication/273656601

\title{
Épisodes d'inactivité et revenus criminels dans une trajectoire de délinquance
}

Article in Canadian Journal of Criminology and Criminal Justice/La Revue canadienne de criminologie et de justice pénale · October 2014

DOI: 10.3138/CJCCJ.2013.F02

CITATIONS

2 authors, including:

Frédéric Ouellet

Université de Montréal

44 PUBlications 169 CITATIONS

SEE PROFILE

Some of the authors of this publication are also working on these related projects:

Project Trajectories of women victims of intimate partner violence / Les trajectoires de femmes victimes de violence conjugale View project

Project $\quad$ Trajectoires de joueurs excessifs/pathologiques View project
READS

791 


\section{Épisodes d'inactivité et revenus criminels dans une trajectoire de délinquance}

Par

Frédéric Ouellet et Pierre Tremblay,

École de criminologie, Université de Montréal

30 novembre 2013

Correspondre avec : Frédéric Ouellet

frederic.ouellet.1@umontreal.ca

École de criminologie

Université de Montréal

C.P. 6128, succursale Centre-ville

Montréal QC H3C 3J7

Canada

**post-print version; Published source : Ouellet, F. \& Tremblay, P. (2014). Épisodes d'inactivité et revenus criminels dans une trajectoire de délinquance. Canadian journal of criminology and criminal justice/Revue canadienne de criminologie et de justice pénale, 56(5): 527-562.

Link to publisher version: http://www.utpjournals.press/doi/abs/10.3138/CJCCJ.2013.F02

Les auteurs voudraient remercier Chloé Leclerc et Rémi Boivin pour les précieux commentaires émis sur les versions préliminaires de cet article. Les auteurs tiennent également à remercier les évaluateurs de la Revue canadienne de criminologie et de justice pénale ainsi que son éditeur adjoint pour leurs commentaires sur la version préliminaire de cet article.

***Les auteurs de cette étude intitulée Épisodes d'inactivité et revenus criminels dans une trajectoire de délinquance attestent que ni l'article présenté, ni une version de cet article, n'ont été publiés, et qu'ils ne sont pas considérés pour publication ailleurs. 


\title{
RÉSUMÉ
}

L'instabilité de l'activité criminelle dans le temps est déjà bien documentée. On connaît toutefois peu les circonstances qui expliquent ces variations à court terme. Une meilleure connaissance de ces facteurs est souhaitable puisqu'il est possible que les transitions et les changements à court terme précèdent les points tournants des carrières criminelles. Les conditions qui rendent compte d'une interruption temporaire des activités peuvent, par exemple, contribuer à expliquer un désistement définitif. L'étude se fonde sur les trajectoires de 172 délinquants impliqués dans des crimes à but lucratif et analyse les variations mensuelles de leurs revenus criminels ainsi que les épisodes d'inactivité criminelle à l'intérieur d'une période fenêtre de 36 mois. La méthode des calendriers d'histoire de vie combinée aux modèles hiérarchiques permet d'examiner conjointement le rôle de facteurs statiques (les caractéristiques individuelles des sujets) et dynamiques (les circonstances de vie). Les résultats mettent en évidence l'importance des événements qui marquent le style de vie des délinquants et des paramètres qui caractérisent l'engagement criminel dans la compréhension des variations dans les trajectoires à l'étude. Ils soulignent également l'importance de la finalité derrière les activités criminelles pour expliquer la décision des délinquants de cesser temporaire leurs activités illicites.

Mots clés : performance criminelle, épisode d'inactivité criminelle, carrière criminelle, circonstances de la vie conventionnelle et délinquante.

\begin{abstract}
The instability of the criminal activity over time is already well documented. However, little known on the circumstances that can explain these short-term variations. It is possible that transitions and changes in short-term precede turning points in criminal careers. For example, conditions that account for a temporary interruption of criminal activities might help to explain a more definitive desistance from crime. It appears therefore appropriate to improve our knowledge on these factors. The study is based on the trajectories of 172 offenders' involved lucrative forms of crime. It focuses on changes in criminal earnings and episodes of temporary desistance within a window period of 36 months. The method of life history calendars combined with hierarchical models allows to analyze the role of static determinants (individual characteristics) and dynamic (life circumstances) in understanding variations in criminal activity on a monthly basis. Results highlight the weight of events that mark offenders' lifestyle and also the parameters that characterize criminal involvement in predicting variations in trajectories observed. They also emphasize the importance of criminal achievement in explaining the decision of offenders to stop temporarily their illegal activities.
\end{abstract}

Keywords: criminal achievement, episodes of criminal inactivity, criminal career, conventional and deviant life circumstances. 


\section{INTRODUCTION}

La dynamique des carrières et des trajectoires criminelles est complexe et sa compréhension passe par plusieurs dimensions. Bon nombre d'études se sont intéressées à la performance criminelle. Celle-ci est généralement mesurée par les gains que retirent les délinquants de leurs activités criminelles (McCarthy et Hagan, 2001; Morselli et Tremblay, 2004; Morselli, Tremblay et McCarthy, 2006; Robitaille, 2004; Uggen et Thompson, 2003; Wilson et Abrahamse, 1992). On peut aussi la mesurer, quoique moins fréquemment, par l'impunité, la capacité et l'habileté à éviter les sanctions pénales (Chaiken et Chaiken, 1985; Johnson, Natarajan et Sanabria, 1993; Kazemian et Le Blanc, 2007). Ces études tiennent pour acquis que la performance criminelle est stable au fil du temps, alors que ce n'est souvent pas le cas. Elles tracent un portrait statique du phénomène qui sous-estime nécessairement les causes situationnelles et les circonstances de vie dans lesquelles évoluent les délinquants.

\section{La performance criminelle}

Comprendre la dynamique derrière le succès (ou l'échec) que connaissent les délinquants dans leur carrière criminelle peut non seulement contribuer aux connaissances sur les modèles de réussites (qui réussit?), mais aussi sur le contexte favorable à la réussite (quand réussit-on le mieux?). L'étude de la performance criminelle permet aussi de mieux comprendre des autres dimensions des carrières criminelles. Shover et Thompson (1992) ont montré l'existence d'un lien robuste entre les succès passés à éviter les sanctions et le désistement : ceux qui ont du succès sont moins enclins à cesser leurs activités. Il pourrait en être de même pour les revenus criminels. On peut penser que le montant des gains soutirés illégalement incite le criminel à poursuivre ses activités et ce momentum de ses succès ponctuels l'encourageant à augmenter la fréquence de ses crimes. L'inverse est aussi vrai : des revenus modestes ou décevants peuvent le décourager et l'inciter à penser que le jeu n'en vaut pas la chandelle. Cette étude s'intéresse aux variations temporelles des revenus criminelles, mais aussi au lien entre la performance criminelle et la décision d'interrompre temporairement les activités criminelles.

L'étude des revenus criminels est importante, surtout si l'on considère que la plupart des crimes commis sont motivés par l'appât du gain (Uggen et Thompson, 2003). Que sait-on à propos des revenus criminels? Les études de Levitt et Venkatesh (2000) et celle de Wilson et Abrahamse (1992) indiquent que les modèles de réussites sont rares dans les milieux criminels. En fait, la plupart des recherches sur le sujet soulignent l'existence d'importantes variations quant à la performance criminelle. De nombreux délinquants retirent des revenus de leurs crimes (McCarthy et Hagan, 2001; Morselli et Tremblay, 2004; Morselli, Tremblay et McCarthy, 2006; Robitaille, 2004; Tremblay et Morselli, 2000). Mais l'analyse détaillée de Morselli et Tremblay (2010) des revenus criminels de l'enquête utilisée dans le cadre de cette étude souligne l'asymétrie de leur répartition. De la somme totale d'environ 120 millions de dollars déclarée par les répondants durant la période à l'étude, le $10 \%$ des délinquants qui retirent davantage du crime rapporte à lui seul $69 \%$ de cette somme totale, si on élargit le spectre au $20 \%$ des délinquants les plus performants, ceux-ci cumulent $84 \%$ du total des gains criminels. Qu'est-ce qui différencie les délinquants qui réussissent de ceux qui ne réussissent pas?

À partir d'un échantillon de 376 jeunes âgés de 24 ans ou moins de la région de Toronto, McCarthy et Hagan (2001) ont mis en évidence l'importance du capital humain, social, mais 
surtout du capital personnel - le désir de s'enrichir, une propension aux risques, un désir de coopérer et les compétences à la réussite générale - dans la réussite criminelle. Ils ont également montré qu'il existait un parallèle entre les aptitudes liées à la prospérité légitime et illégitime. Les études réalisées par Morselli et Tremblay (2004), Morselli, Tremblay et McCarthy (2006) se sont basées sur un sondage effectué auprès de détenus incarcérés dans des pénitenciers fédéraux. La première étude souligne l'importance de certains traits de personnalité (le manque de contrôle de soi) et du capital social (la taille des réseaux criminels) dans la réussite criminelle. La seconde enquête souligne l'apport des mentors dans les carrières criminelles, l'apprentissage auprès d'un mentor a pour effet d'augmenter les revenus criminels et de diminuer les risques d'arrestation. Un résultat apparemment contre-intuitif de l'étude de Morselli et Tremblay (2004) était que le manque de contrôle de soi, un trait de personnalité qui combine présentisme, impulsivité et goût du risque et qui devait prédire la délinquance, prédisait aussi le degré de réussite criminelle. Une analyse plus fine indiquait que cet effet était plus prononcé lorsqu'il s'agissait de délinquance contre les biens que de délinquance de trafic. Nous examinerons l'hypothèse selon laquelle l'impulsivité et la témérité pourrait expliquer la volatilité ou l'instabilité des épisodes de réussite dans une trajectoire de délinquance. La réanalyse du sondage de la Rand Corporation effectuée par Robitaille (2004) montre que les gains criminels sont favorisés par les aptitudes stratégiques que déploient les délinquants, la spécialisation, l'estime de soi et l'absence d'une consommation abusive d'alcool durant la période de référence. Deux facteurs antécédents contribuent aussi à majorer les chances de réussite criminelle : l'âge et le nombre de condamnations antérieures. Selon Robitaille, ces deux facteurs font preuve de l'accumulation du capital humain et social. Autrement dit, la maturation et les expériences d'incarcération incitent ces délinquants à acquérir des compétences qui majorent leurs chances de réussite criminelle.

\section{L'instabilité de l'activité criminelle}

Matza (1967) a observé, il y a longtemps, que les contrevenants ne commettaient pas constamment des crimes (voir Adler et l'Adler, 1983, mais aussi Nagin et Land, 1993), qu'ils étaient enclins à se désister temporairement. Ce parcours en «zigzag », composé de cycles épisodiques d'activité criminelle et d'abstinence, serait caractéristique de la majorité des trajectoires de délinquance (voir Laub et Sampson, 2003; Piquero, 2004; Horney et Marshall, 1991). L'instabilité des revenus criminels est elle aussi un fait bien établi dans la littérature (Hagan et McCarthy, 1997;). La volatilité des gains criminels oblige plusieurs délinquants à occuper des emplois légitimes, que ce soit sur une base permanente ou temporaire (Hagan et McCarthy, 1997; Uggen et Thompson, 2003; Morselli et Tremblay, 2004; Thompson et Uggen, 2012). On connait assez mal les éléments qui permettent d'expliquer et de comprendre ces épisodes d'intermittence (Piquero, 2004).

Quatre études (cf. Tableau 1) se sont intéressées aux changements à court de la cadence délictuelle (Horney, Osgood et Marshall, 1995; Griffin et Armstrong, 2003), du penchant des délinquants à diversifier leurs activités (McGloin, Sullivan, Piquero et Pratt, 2007) et de leurs revenus criminels mensuels (Uggen et Thompson, 2003). La cadence délictuelle (lambda) mensuelle et le montant des revenus criminels diminueraient pendant les mois de cohabitation conjugale. Horney, Osgood et Marshall (1995) sont plus nuancés. Ils montrent que les effets de cohabitation matrimoniale et conjugale varient en fonction du type de crime commis. Ainsi, il est moins probable que les délinquants commettent des voies de fait les mois où ils sont en couple, 
alors que, pour les crimes liés à la drogue, c'est l'inverse : les délinquants semblent s'y adonner davantage durant les épisodes de vie conjugale. Horney et coll. (1995) ainsi que McGloin et coll. (2007) montrent que la participation et la diversification ne sont pas influencées par la situation d'emploi des délinquants. Uggen et Thompson (2003; voir aussi Uggen, 2000) montrent, quant à eux, que les contrevenants augmentent leurs revenus criminels pendant les mois de chômage.

Ces auteurs ne se sont pas limités aux fluctuations des pressions des mécanismes informels de régulation sociale (emploi, mariage), mais aux fluctuations de circonstances associées au style de vie des délinquants. Horney et coll. (1995) constatent que les mois où les contrevenants augmentent leur consommation d'alcool et de drogue, ils augmentent également la probabilité de commettre des délits. Les résultats des études d'Uggen et Thompson (2003) et de McGloin et coll. (2007) indiquent qu'une hausse de la consommation de drogue augmente les revenus criminels et la diversification des activités criminelles. Uggen et Thompson (2003) montrent aussi que leurs revenus criminels diminuent lorsque les délinquants jugent que leurs risques d'arrestation sont plus élevés. On peut supposer que ces risques seront plus importants durant les épisodes de surveillance pénale. Si Horney et ses collègues (1995) indiquent que les épisodes de probation n'ont aucun effet sur la participation criminelle, l'étude de McGloin et coll. (2007) aboutit à une conclusion contraire : dans de telles circonstances, les contrevenants restreignent, sans les arrêter complètement, le nombre d'activités dans lesquelles ils sont impliqués. Autrement dit, les risques de sanction, qu'ils soient objectifs ou subjectifs, ne poussent pas les criminels à l'abstinence, mais les incitent à la prudence et à la modération.

Tableau 1 : Synthèse des études sur les variations temporelles à court terme de l'activité criminelle

\begin{tabular}{|c|c|c|c|c|c|c|c|c|}
\hline Auteurs & Année & Échantillon & Méthode & $\begin{array}{l}\text { Dimension de } \\
\text { la carrière cri. }\end{array}$ & $\begin{array}{c}\text { Variable } \\
\text { dépendante }\end{array}$ & $\begin{array}{l}\text { Caract. } \\
\text { indiv. }\end{array}$ & $\begin{array}{l}\text { Circ. } \\
\text { de vie }\end{array}$ & Principaux résultats \\
\hline $\begin{array}{l}\text { Horney, } \\
\text { Osgood \& } \\
\text { Marshall }\end{array}$ & 1995 & $\begin{array}{l}658 \text { criminels } \\
\text { nouvellement } \\
\text { condamnés }\end{array}$ & $\begin{array}{l}\text { Calendriers } \\
\text { d'histoire } \\
\text { de vie }\end{array}$ & $\begin{array}{l}\text { Fréquence des } \\
\text { crimes commis }\end{array}$ & $\begin{array}{l}\text { Probabilités de } \\
\text { s'engager/arrêter } \\
\text { dans quatre formes } \\
\text { de crime }\end{array}$ & NON & OUI & $\begin{array}{l}\text { Changements à court terme } \\
\text { sont fortement liés aux } \\
\text { circonstances de vie } \\
\text { conventionnelles }\end{array}$ \\
\hline $\begin{array}{l}\text { Griffin \& } \\
\text { Armstrong }\end{array}$ & 2003 & $\begin{array}{l}195 \text { femmes en } \\
\text { probation avec } \\
\text { problèmes de } \\
\text { consommation }\end{array}$ & $\begin{array}{l}\text { Calendriers } \\
\text { d'histoire } \\
\text { de vie }\end{array}$ & $\begin{array}{l}\text { Fréquence des } \\
\text { crimes commis }\end{array}$ & $\begin{array}{l}\text { Probabilités de } \\
\text { s'engager dans } \\
\text { deux formes de } \\
\text { crime }\end{array}$ & NON & OUI & $\begin{array}{l}\text { Dynamique de l'activité } \\
\text { criminelle est influencée par } \\
\text { la nature des crimes et les } \\
\text { circonstances de vie }\end{array}$ \\
\hline $\begin{array}{l}\text { Uggen, \& } \\
\text { Thompson }\end{array}$ & 2003 & $\begin{array}{l}\text { Programme de } \\
\text { réinsertion au } \\
\text { travail : } \\
2268 \text { criminels, } \\
1394 \text { dépendants } \\
\text { aux drogues, } \\
1241 \text { décrocheurs }\end{array}$ & $\begin{array}{l}\text { Design } \\
\text { longitudinal } \\
\text { (suivi entre } \\
18 \text { et } 36 \\
\text { mois) }\end{array}$ & $\begin{array}{l}\text { Performance } \\
\text { criminelle }\end{array}$ & $\begin{array}{l}\text { Variations } \\
\text { mensuelles des } \\
\text { gains criminelles }\end{array}$ & NON & OUI & $\begin{array}{l}\text { Revenus criminels sont } \\
\text { sensibles aux épisodes } \\
\text { d'emploi, les relations } \\
\text { familiales, l'expérience } \\
\text { criminelle, et les risques } \\
\text { perçus et les avantages } \\
\text { inhérents à la criminalité }\end{array}$ \\
\hline $\begin{array}{l}\text { McGloin, } \\
\text { Sullivan, } \\
\text { Piquero \& } \\
\text { Pratt }\end{array}$ & 2007 & $\begin{array}{l}658 \text { criminels } \\
\text { nouvellement } \\
\text { condamnés } \\
\text { (même que celui } \\
\text { utilisé par de } \\
\text { Horney et coll.) }\end{array}$ & $\begin{array}{l}\text { Calendriers } \\
\text { d'histoire } \\
\text { de vie }\end{array}$ & $\begin{array}{l}\text { Diversification } \\
\text { de l'activité } \\
\text { criminelle }\end{array}$ & Index de diversité & OUI & OUI & $\begin{array}{l}\text { À la fois la propension } \\
\text { individuelle et les } \\
\text { circonstances de vie } \\
\text { conventionnelles influencent } \\
\text { les épisodes de } \\
\text { spécialisation/diversification }\end{array}$ \\
\hline
\end{tabular}

Bien que forts instructives, ces recherches, à une exception près, accordent peu d'importance aux caractéristiques individuelles des délinquants. Dans la perspective du «life course », ce sont les circonstances de vie qui ont une influence directe sur les opportunités criminelles qui se présentent aux individus (Horney et coll., 1995; Sampson et Laub, 1990, 1993; Laub et Sampson, 2003; Griffin et Armstrong, 2003; Farrington, 2005 ; McGloin et coll., 2007) et non une prédisposition générale. La perspective développementale propose qu'une prédisposition générale, un trait qui est stable au fil du temps, motive les délinquants à commettre des délits 
(Gottfredson et Hirschi, 1990; Gottfredson, 2005). Les individus marqués par cette propension aux crimes sont davantage attirés par les opportunités criminelles et sont davantage à leur recherche (Piquero et Tibbetts, 1996; Pratt et coll., 2005). L'étude de McGloin et coll. (2007) montre que la précocité de la trajectoire de délinquance influence à long terme le penchant à la diversification des activités criminelles (laquelle peut favoriser dans certaines circonstances leurs revenus). En revanche, Kazemian et Le Blanc (2007) ont tenté de prédire le succès à éviter les appréhensions policières à l'aide d'une étude longitudinale prospective. À partir d'un échantillon de jeunes judiciarisés de la région de Montréal rencontré à l'adolescence (âgés de 13 à 17 ans), mais aussi à 20 ans, à 30 ans et à 40 ans, ils concluent qu'il est particulièrement difficile de prédire ce type de succès à l'âge adulte à partir des mesures prises à l'adolescence. Ces auteurs attribuent ce résultat aux variations des opportunités criminelles qui existent entre les individus.

\section{La présente étude}

Cette étude utilise un modèle d'analyse dynamique et hiérarchique pour spécifier les déterminants statiques et dynamiques des épisodes plus on moins prolongés d'activités criminelles et la manière dont s'intercalent les séquences d'inactivité dans le crime. Nous débuterons avec l'examen des mois d'activités criminelles et utiliserons les revenus criminels mensuels comme un indicateur de leur performance individuelle. Nous analyserons ensuite les éléments qui incitent certains contrevenants à interrompre (ou non) leurs activités illicites durant la période fenêtre et jusqu'à quel point cette décision est influencée par leur performance. Les modèles hiérarchiques sur les carrières criminelles examinent les changements intra-individuels en prenant soin de contrôler les différences entre les délinquants.

Ces modèles ont aussi une deuxième vertu. Ils accordent à chaque individu l'opportunité de décider les variations de leurs revenus criminels qu'ils jugent significatives (chaque individu possède sa propre constante), et des événements de vie qu'ils considèrent comme marquants pour eux - libération conditionnelle, séparation ou rencontre, retour sur le marché du travail. Par exemple, si le délinquant $\mathrm{A}$ augmente ses revenus criminels mensuels de $500 \$$ à $1000 \$$ et que le délinquant B augmente les siens de 2000 \$ à 4000 \$, le changement de revenu est aussi significatif pour A que B. On peut différencier les délinquants selon leur performance, mais les moins performants comme les plus performants peuvent tous deux connaître des hausses ou des baisses de leurs revenus et en tirer des conséquences sur leur décision ou non de se désister. Si le délinquant $\mathrm{B}$ voyait ses revenus mensuels chuter à 1,000 \$, il serait peut-être incité à envisager l'option de cesser ses activités criminelles. Pour le délinquant A, de tels revenus pourraient au contraire l'encourager à « continuer». Nous analyserons les trajectoires criminelles de 172 détenus. La période examinée se restreint aux 36 mois précédant l'incarcération que les détenus purgeaient au moment de l'entretien. L'échantillon est constitué de délinquants adultes impliqués dans une criminalité lucrative (crime contre la propriété et crime de marché) et bien enracinée dans les milieux criminels.

\section{DONNÉES ET STRATÉGIE D’ANALYSE}

Les données de cet article proviennent d'entretiens réalisés entre 2000 et 2001 auprès de 262 détenus rencontrés dans cinq pénitenciers à proximité de Montréal. Cette enquête se centrait exclusivement sur les délinquants impliqués dans une criminalité lucrative. Il importe de 
mentionner que ce sondage a déjà été analysé antérieurement pour montrer les bénéfices du manque de contrôle de soi (Morselli et Tremblay, 2004) et aussi pour examiner l'impact des mentors (Morselli, Tremblay et McCarthy, 2006) et de la classe sociale (Charest et Tremblay, 2009) sur la réussite criminelle, mais aussi pour explorer le lien entre les risques d'arrestation et la taille des marchés de drogues illicites (Bouchard et Ouellet, 2011). Toutefois, les calendriers d'histoire de vie qui avaient été incorporés dans cette enquête n'ont jamais été exploités à leur plein potentiel. Aucune des études rapportées ne s'est penchée sur les variations mensuelles de l'activité criminelle au sein des trajectoires individuelles. La contribution de la présente recherche est qu'elle compare les délinquants par rapport à eux-mêmes et examine l'influence des circonstances de vie sur leur comportement durant les 36 mois de leur trajectoire de délinquance.

Les détenus interviewés étaient incarcérés dans des établissements à sécurité moyenne et minimale. Les entretiens réalisés en face à face ont été privilégiés. Contrairement à d'autres sondages faits auprès de détenus, les questionnaires n'ont pas été auto-administrés (Peterson et Braiker, 1981; Chaiken et Chaiken, 1982; Horney, Osgood et Marshall 1995). Le recrutement des détenus a été fait à partir des listes des populations carcérales présentes lors des entretiens. Chaque intervieweur s'est fait assigner un groupe de vingt à trente détenus de façon aléatoire. Après l'arrivée du groupe de recherche (3-5 intervieweurs), nous avons procédé à l'appel par interphone d'un détenu à la fois, pour solliciter la participation volontaire des sujets à notre sondage. Les détenus n'ont pas été rémunérés pour leur participation. $35 \%$ des détenus n'étaient pas disponibles lors de l'appel. La proportion des sujets qui ont accepté de participer à ce sondage est de 80 \%. La durée moyenne des entretiens était d'une heure et demie. Pour faciliter la mémorisation des événements survenus durant la période fenêtre, celle-ci correspond aux 36 mois précédents l'incarcération actuelle. Les détenus incarcérés depuis plus de sept ans ont été exclus. Par conséquent, la majorité des détenus (76\%) étaient incarcérés depuis moins de 2 ans. On trouvera une présentation détaillée des procédures de cueillette utilisées de cette enquête dans Charest (2004) et dans Morselli et Tremblay (2010).

Bien que 262 détenus aient été rencontrés durant la phase de recrutement, l'échantillon utilisé dans cet article se compose de 172 détenus. Nous avons exclu les détenus qui n'ont rapporté aucune activité criminelle lucrative durant la période fenêtre de 36 mois $(n=45)$ ainsi que les détenus dont les activités criminelles se sont échelonnées sur moins de trois mois durant la même période $(n=22)$. On doit compter sur au moins trois temps de mesure afin d'examiner les trajectoires de développement. Il s'agit d'un minimum nécessaire à l'étude du changement (Dupéré et coll., 2007). Les données manquantes dans les calendriers ont également occasionné le retrait de 23 participants; la stratégie d'analyse choisie dans cette étude supprime les cas qui présentent des données manquantes. La fiabilité des données issues de la période fenêtre est facilitée par la stratégie développée par Freedman, Thornton, Camburn, Alwin, et YoungDeMarco (1988) qui utilisent des calendriers détaillés des circonstances de vie afin d'examiner la dynamique qui entoure les activités criminelles (Ahn, Blumstein et Schervish, 1990; Bradburn, Lance et Shevell, 1987; Griffin et Armstrong, 2003; Horney et Marshall, 1991; Horney et coll., 1995; MacKenzie et Li, 2002; Uggen et Thompson 2003). Bien avant de recueillir l'information relative aux activités criminelles et aux bénéfices qui en résultent, il a été important de mettre en contexte, pour chaque participant, les événements et les circonstances qui sont survenus durant la période fenêtre. Les répondants ont donc été appelés à situer leurs activités temporellement : à chaque mois et dans l'ordre : 1) les mois passés en prison, en probation, en libération conditionnelle ou en maison de transition; 2) les mois durant lesquels ils ont fait l'objet d'une 
arrestation; 3) le lieu de résidence (la ville); 4) les événements de vie (hospitalisation, divorce, naissance d'un enfant, mortalité d'un proche, perte d'emploi); 5) les circonstances de la vie conventionnelle (le détail des emplois occupés, les prestations gouvernementales, les mois passés en couple). Le rappel des expériences criminelles passées et des revenus retirés est facilité une fois que le participant visualise les autres éléments compris dans les calendriers. Ces techniques de remémoration, comme celle utilisée dans cette étude, s'avèrent efficaces pour récupérer les détails ou les informations sur des événements passés. Fischer et coll. (1989) montrent que les victimes et les témoins d'acte criminel donnent davantage de précisions sur leur expérience lorsqu'ils sont soumis à une méthode qui vise à reconstruire le contexte et les circonstances qui entourent le crime.

Les mois de la trajectoire de délinquance des sujets qui ont été sélectionnés devaient satisfaire à deux conditions : 1) une participation à des activités criminelles de trois mois ou plus; 2) un contexte favorable ou qui ne contraint pas l'activité criminelle. Pour examiner la dynamique des épisodes d'inactivité criminelle, nous utilisons uniquement les mois où les délinquants sont en liberté et nous excluons les mois passés en prison (172 participants $\times 30,2$ mois). Pour analyser les changements de revenus, la sélection des mois éligibles est différente (172 sujets x 19,3 mois). Les mois sélectionnés sont les mois ils sont non seulement en liberté, mais aussi actifs. On concède volontiers que les sujets peuvent participer à des activités délinquantes en prison (et qu'ils peuvent éventuellement être accusés de leurs infractions), qu'ils peuvent « travailler » et recevoir un supplément de revenu « légitime », que leur emprisonnement peut avoir un impact sur leurs relations conjugales ou que leur trajectoire de délinquance en prison peut elle-même fluctuer et connaître des périodes d'activité ou de d'inactivité. Cela dit, l'emprisonnement est un «monde à part » qui redéfinit les relations d'emploi, les conditions de rémunération, le répertoire des «infractions », le statut des délinquants et les relations maritales. Nos analyses se limitent à la dynamique des changements de délinquants durant leurs mois de liberté, mais il pourrait être intéressant éventuellement d'élargir cette perspective et de procéder à l'étude dynamique des circonstances de vie susceptibles d'influencer la trajectoire des détenus durant leur incarcération.

\section{Les revenus criminels mensuels et les mois d'inactivité}

Pour mesurer les performances criminelles, nous utilisons les logarithmes des revenus criminels mensuels, car la distribution des revenus est log-normale. Elle permet aussi de neutraliser les effets de vantardise potentiels (Wilson et Abrahamse, 1992). Les délinquants peuvent être actifs, mais ne retirer aucun gain de leurs délits (délit « raté » ou « tentative »; délit interrompu pour cause d'arrestation; délit suivi de vol de la part d'un des partenaires, etc.). Ces mois sont rares (3\% des mois éligibles), mais ils sont incorporés dans l'analyse. Les revenus criminels mensuels moyens pendant les mois d'activité étaient d'environ 7000 (moyenne géométrique $=6843,80 \$$ ). Lorsque l'on étend les revenus mensuels sur l'ensemble des 36 mois constituant la période, la moyenne des revenus mensuels tombe à environ $4000 \$$ par mois. Les mois d'inactivité criminelle sont les mois durant lesquels les détenus étaient libres de commettre des crimes, mais ont décidé, pour de nombreuses raisons, de s'abstenir. La majorité des délinquants de notre échantillon (62\%) ont connu au moins un épisode d'inactivité criminelle durant la période fenêtre. La durée moyenne de cet épisode est de onze mois.

Les modèles linéaires hiérarchiques permettent d'inclure à la fois des indicateurs dynamiques (niveau 1) et statiques (niveau 2) pour expliquer la performance criminelle mensuelle et les 
épisodes d'inactivité. Nous entendons par facteurs dynamiques les variables indépendantes qui varient en fonction du temps; sur une base mensuelle. Les facteurs suivants peuvent être analysés en tant que facteurs dynamiques : la cohabitation conjugale et les revenus légitimes (revenus d'emploi et prestations gouvernementales); la surveillance pénale (libération conditionnelle, probation et maison de transition); le succès passé à éviter les contacts policiers; la diversification des activités criminelle comme une mesure de l'accès aux opportunités criminelles. Les facteurs statiques sont, quant à eux, l'âge au début de la période fenêtre, l'âge au premier crime, le score à l'échelle du manque de contrôle de soi, le niveau de scolarité et le nombre de partenaires qui composent le réseau criminel. Les données de calendrier, dans ce sondage, ne fournissent aucun renseignement quant à la consommation mensuelle d'alcool et de drogue. Bien qu'il ait été préférable d'avoir les informations sur les habitudes de consommation sur une base mensuelle, il s'agit d'une limite, l'indicateur disponible sur ce sujet reflète les habitudes de consommation moyenne durant la période de 3 ans. Il a été demandé aux détenus de qualifier leur consommation moyenne durant la période fenêtre (jamais consommé, quelquefois par mois, quelques fois par semaine, chaque jour ou presque chaque jour, plus d'une fois par jour).

\section{Les facteurs dynamiques : les circonstances de vie}

Une proportion significative des détenus interrogés a bénéficié de revenus d'emploi ou autres revenus légitimes durant la période fenêtre. Les revenus légitimes incluent à la fois les revenus conventionnels issus du marché du travail et les autres formes de revenus légitimes comme les prestations gouvernementales. La moyenne des revenus légitimes mensuels n'est pas élevée dans cet échantillon; moyenne géométrique de 503 \$. La proportion moyenne des mois passés en cohabitation conjugale est de $43 \%$. La proportion des délinquants sous surveillance (libération conditionnelle, probation ou maison de transition) dans cette étude est de 38,4\% et la durée moyenne de la surveillance est de 10 mois, avec un écart-type de 6,3. Pour les délinquants sous surveillance durant la période fenêtre, la surveillance pénale représente en moyenne $20 \%$ des mois passés en liberté, excluant ainsi les mois en prison. Il est aussi intéressant de s'attarder à l'impact de la surveillance, à savoir l'impact de ces épisodes sur la motivation à se désister de ses activités criminelles ou, au contraire, à persister dans le crime.

Tableau 2 : Statistiques descriptives des facteurs dynamiques et statiques pour les modèles de la performance criminelle et des épisodes d'inactivité dans le crime.

\begin{tabular}{|c|c|c|c|c|}
\hline \multirow[b]{2}{*}{ Niveau 1 : Caractéristiques dymaniques } & \multicolumn{2}{|c|}{$\begin{array}{l}\text { Les mois d'activités } \\
\text { criminelles }(\mathrm{N}=3321) \\
\text { (performance criminelle) }\end{array}$} & \multicolumn{2}{|c|}{$\begin{array}{l}\text { Les mois de liberté } \\
\quad(\mathrm{N}=5125) \\
\text { (inactivité criminelle) }\end{array}$} \\
\hline & Moyenne & Écart-type & Moyenne & Écart-type \\
\hline $\begin{array}{l}\text { Inactivité criminelle } \\
(1=\text { oui; } 0=\text { non })\end{array}$ & & & 0,35 & 0,48 \\
\hline $\begin{array}{l}\text { Revenus criminels }(\ln ) \\
\text { (moyenne géométrique) }\end{array}$ & $\begin{array}{c}8,83 \\
(6843,80 \$)\end{array}$ & 2,23 & $\begin{array}{c}5,74 \\
(311,06 \$)\end{array}$ & 4,58 \\
\hline $\begin{array}{l}\text { Cohabitation conjugale }(\%) \\
(1=\text { oui; } 0=\text { non })\end{array}$ & 0,43 & 0,50 & 0,42 & 0,49 \\
\hline $\begin{array}{l}\text { Revenus légitimes }(\mathrm{ln}) \\
\text { (moyenne géométrique) }\end{array}$ & $\begin{array}{c}6,22 \\
(502,70 \$)\end{array}$ & 3,36 & $\begin{array}{c}6,12 \\
(454,87 \$)\end{array}$ & 2,87 \\
\hline $\begin{array}{l}\text { Surveillance pénale }(\%) \\
(1=\text { oui; } 0=\text { non })\end{array}$ & 0,20 & 0,19 & 0,21 & 0,41 \\
\hline
\end{tabular}


Succès passé à éviter les contacts policiers (\%)

(mois sans contact policier/mois d'activités criminelles)

Diversification

(nombre d'activités criminelles distinctes)

Niveau 2 : Caractéristiques statiques $(\mathrm{N}=172)$

Âge

(au début de la période fenêtre)

Niveau d'éducation (\%)

( $1=$ diplôme d'études secondaires; $0=$ sans diplôme sec.)

Précocité de la carrière criminelle

(l'âge lors du premier crime)

Score à l'échelle du manque de contrôle de soi

(somme des 24 items de Grasmick et coll. (1993))

Consommation quotidienne d'alcool (\%)

(1=oui; $0=$ non)

Utilisation mensuelle de drogues dures (\%)

(1=oui; $0=$ non)

Réseau criminel

(nombre de partenaires dans le réseau criminel)

\begin{tabular}{ll|ll}
0,88 & 0,27 & 0,71 & 0,41 \\
1,82 & 1,04 & \\
32,37 & 8,51 & \\
0,19 & 10,53 & \\
15,20 & 6,81 & \\
80,28 & 17,14 & \\
0,28 & 0,45 & \\
0,58 & 0,50 & \\
11,02 & 10,83 &
\end{tabular}

Les contacts auto-révélés avec les forces de l'ordre incluent les avertissements, les contrôles d'identité, les interrogatoires et les arrestations. Les contacts avec les policiers mesurent la capacité ou le succès des délinquants à éviter les appréhensions policières. Les modèles hiérarchiques basés sur des données rétrospectives des carrières criminelles sont bien adaptés pour étudier la capacité des délinquants à éviter l'arrestation (Jacobs, 1996 ; Stafford et Warr, 1993). Cette expérience de l'impunité a pour effet de façonner leurs évaluations des risques auxquels leurs activités illicites les exposent. On trouvera dans Stafford et Warr, un effort important pour intégrer dans une théorie de la dissuasion générale et spécifique, les expériences directes ou indirectes d'impunité (Stafford et Warr, 1993; voir aussi Piquero et Paternoster, 1998; Piquero et Pogarsky, 2002). Shover et Thompson (1992) montrent notamment dans leur étude que les décisions des délinquants de se désister ou de persévérer dans leurs activités criminelles dépendent de leur expérience d'impunité. Pour mesurer le sentiment d'impunité des sujets nous avons procédé de la manière suivante : si un délinquant est actif dix mois avant d'être appréhendé pour la première fois par la police, son succès relatif à éviter l'arrestation est de $100 \%$ le mois avant d'être arrêté (10 sur 10) mais de $90 \%$ le mois suivant ( 9 sur 10). Après ce premier contact, s'il continue ses activités criminelles durant cinq mois avant d'être arrêté de nouveau, sa capacité à éviter les contacts policiers diminue à $87 \%$ (13 sur 15). Le succès moyen à éviter les appréhensions dans notre échantillon est de $88 \%$, avec un écart-type de $27 \%$.

Pour capturer les variations mensuelles des occasions criminelles, nous avons analysé les variations du nombre d'activités illégales dans lesquelles les contrevenants sont mensuellement impliqués. Les détenus qui ont participé au sondage devaient énumérer la variété des secteurs d'activité auxquels ils avaient participé durant la période fenêtre (types de marchés criminels et types de crimes contre les biens) ${ }^{1}$. Durant les mois d'activité criminelle, les contrevenants ont en

\footnotetext{
${ }^{1}$ Les différents secteurs d'activités criminelles comprennent : vol qualifié, cambriolage, vol d'auto, fraude escroquerie/délinquance d'affaire, autres crimes contre les biens, vente de drogue et distribution, contrebande, prêt usuraire, marché relié au sexe, paris illégaux, recel de biens volés, autre délinquance de marché.
} 
moyenne été impliqués dans deux secteurs d'activités criminelles différents (moyenne = 1,82; écart-type $=1,04)$.

\section{Les facteurs statiques : caractéristiques des participants}

Les participants de cette étude ont complété l'échelle du manque de contrôle de soi de Grasmick et coll. (1993). Le score moyen à cette échelle est de 80,28, avec un écart-type de 14,5. Le manque de contrôle de soi tient compte d'une série de traits qui sont corrélés à la propension criminelle. La méta-analyse de Pratt (2000) suggère que cette échelle est valide. Morselli et Tremblay (2004) montrent qu'une forte propension criminelle, un score élevé à l'échelle du manque de contrôle de soi se transpose par des gains criminels plus élevés, et que l'impulsivité, l'égocentrisme, le goût du risque et la préférence pour des tâches physiques peuvent nuire aux visées légitimes, mais faciliter les visées illégitimes. Nous utilisons le résultat à l'échelle de Grasmick comme un indicateur des différences individuelles au niveau de la propension criminelle, comme une motivation à poursuivre une carrière criminelle sérieuse. L'âge moyen au début de la période fenêtre est de 33 ans, avec un écart-type de 8,5. Dans la mesure où nous contrôlons pour l'impulsivité, le goût du risque et les autres attributs du manque de contrôle de soi, nous considérons que l'âge capture un effet de maturation (Hirschi et Gottfredson, 1983). Le niveau d'éducation peut constituer un atout à la réussite criminelle. Peu des sujets rencontrés ont obtenu un diplôme d'études secondaires. Nous distinguons par conséquent les sujets qui ont leur diplôme secondaire $(18,6 \%)$ de ceux qui ne l'ont pas $(81,6 \%)$. Les délinquants avaient en moyenne15, 2 ans (écart-type $=6,8$ ) lorsqu'ils ont commis leur premier crime. La précocité de la délinquance est un indicateur fiable de la durée de la carrière criminelle. Or, il est difficile de prédire si la précocité se transpose par des gains criminels supérieurs durant la carrière criminelle.

Les participants ont rapporté le niveau d'alcool et de drogues dures consommés durant la période fenêtre. Nous distinguons les participants qui rapportent une consommation quotidienne d'alcool et les utilisateurs de drogues dures (consommation mensuelle de cocaïne, héroïne, crack, etc.). Dans la section du questionnaire sur les contacts, il était demandé aux participants de rapporter le nombre de partenaires, de fournisseurs, de clients, de mentors et autres individus utiles à leurs activités criminelles. Les participants avaient en moyenne onze partenaires dans leur réseau criminel $^{2}$.

\section{La stratégie d'analyse}

Deux modèles sont utilisés dans la présente étude, ceux-ci sont déterminés par la nature des variables dépendantes. Un modèle linéaire hiérarchique, MLH, est utilisé pour explorer la performance criminelle, c'est-à-dire les revenus mensuels du crime. Le MLH est une variante d'un modèle de régression multiple pour données emboitées ou hiérarchisées (Horney et coll., 1995). En plus d'assurer l'indépendance des termes d'erreurs, il nous renseigne sur la variance expliquée par chacun des niveaux d'analyse. Dans la deuxième analyse, nous nous intéressons aux épisodes d'inactivité. Comme l'inactivité dans le crime est mesuré de manière binaire $(0=$ non, $1=$ oui) et que les termes d'erreurs d'une variable binaire ne suivent pas une

\footnotetext{
${ }^{2}$ La consommation d'alcool ou de drogues ainsi que le nombre de partenaires ont tendance à varier dans le temps. Une limite de l'enquête est ne pas avoir incorporé cette consommation dans les calendriers individuels. Elle est donc reléguée aux facteurs statiques.
} 
distribution normale, nous utilisons un modèle linéaire hiérarchique généralisé, abrégé MLHG. Le MLHG est une variante d'un modèle de régression logistique pour des données emboîtées, qui prédit les chances qu'un événement se produise par rapport aux chances qu'il ne se produise pas.

\section{RÉSULTATS}

\section{Les variations mensuelles des gains criminels}

L'utilisation de modèles linéaires hiérarchiques permet de calculer le coefficient de corrélation intra-classe à partir du modèle « inconditionnel » ou «vide » (pour plus de détails, voir Luke, 2004). Le coefficient de corrélation intra-classe renseigne sur la répartition de la variance sur les deux niveaux. Pour les gains criminels, $72,1 \%$ des variations s'expliquent par les circonstances de vie et 27,9\% par les caractéristiques des détenus. La force du coefficient de corrélation intraclasse justifie l'utilisation conjointe d'indicateurs dynamiques et statiques.

Tableau 3 : Les circonstances de vie et les caractéristiques des participants sur les gains criminels mensuels : modèle linéaire hiérarchique, coefficients de régression non standardisés.

\begin{tabular}{|c|c|c|c|}
\hline REVENUS CRIMINELS MENSUELS & $\frac{\text { MODÈLE 1 }}{\begin{array}{c}\text { Contrôles } \\
\text { sociaux }\end{array}}$ & $\frac{\text { MODÈLE 2 }}{\text { Impunité }}$ & $\begin{array}{c}\text { MODÈLE 3 } \\
\begin{array}{c}\text { Diversification } \\
\text { criminelle }\end{array}\end{array}$ \\
\hline \multicolumn{4}{|l|}{ Niveau $1(\mathrm{~N}=3321)$} \\
\hline Cohabitation conjugale & $\begin{array}{l}0,01 \\
(0,09)\end{array}$ & $\begin{array}{l}0,01 \\
(0,09)\end{array}$ & $\begin{array}{l}0,08 \\
(0,08)\end{array}$ \\
\hline Revenu légitime & $\begin{array}{c}-0,04 * * \\
(0,01)\end{array}$ & $\begin{array}{c}-0,04 * * \\
(0,01)\end{array}$ & $\begin{array}{c}-0,02 \\
(0,01)\end{array}$ \\
\hline Surveillance pénale & $\begin{array}{c}-0,72 * * * \\
(0,12)\end{array}$ & $\begin{array}{c}-0,64 * * * \\
(0,11)\end{array}$ & $\begin{array}{c}-0,49 * * \\
(0,11)\end{array}$ \\
\hline Succès passé à éviter les contacts policiers & & $\begin{array}{l}1,18 * * * \\
(0,16)\end{array}$ & $\begin{array}{l}0,98 * * * \\
(0,15)\end{array}$ \\
\hline Diversification criminelle & & & $\begin{array}{l}0,86 * * * \\
(0,05)\end{array}$ \\
\hline \multicolumn{4}{|l|}{ Niveau $2(N=172)$} \\
\hline Âge & $\begin{array}{l}-0,00 \\
(0,02)\end{array}$ & $\begin{array}{l}-0,01 \\
(0,02)\end{array}$ & $\begin{array}{l}-0,00 \\
(0,02)\end{array}$ \\
\hline Niveau de scolarité & $\begin{array}{l}0,43 \\
(0,46)\end{array}$ & $\begin{array}{l}0,34 \\
(0,45)\end{array}$ & $\begin{array}{l}0,40 \\
(0,45)\end{array}$ \\
\hline Précocité de la carrière criminelle & $\begin{array}{l}-0,00 \\
(0,02)\end{array}$ & $\begin{array}{l}-0,01 \\
(0,03)\end{array}$ & $\begin{array}{l}-0,00 \\
(0,03)\end{array}$ \\
\hline Manque de contrôle de soi & $\begin{array}{l}0,02 \\
(0,01)\end{array}$ & $\begin{array}{l}0,02 \\
(0,01)\end{array}$ & $\begin{array}{l}0,01 \\
(0,01)\end{array}$ \\
\hline Consommation quotidienne d'alcool & $\begin{array}{l}0,97 * * \\
(0,39)\end{array}$ & $\begin{array}{l}0,97 * * \\
(0,39)\end{array}$ & $\begin{array}{l}0,98 * * \\
(0,38)\end{array}$ \\
\hline Utilisation de drogues dures & $\begin{array}{c}-0,36 \\
(0,39)\end{array}$ & $\begin{array}{l}-0,33 \\
(0,38)\end{array}$ & $\begin{array}{c}-0,43 \\
(0,38)\end{array}$ \\
\hline Réseau criminel & $\begin{array}{l}0,03 * \\
(0,01)\end{array}$ & $\begin{array}{l}0,03 * \\
(0,01)\end{array}$ & $\begin{array}{l}0,02 \\
(0,01)\end{array}$ \\
\hline
\end{tabular}




\begin{tabular}{llll}
\hline DÉVIANCE & 12205,49 & 12156,48 & 11880,13
\end{tabular}

$*=\mathrm{p}<, 05 ; * *=\mathrm{p}<, 01 ; * * *=\mathrm{p}<, 001$ (les erreurs types sont entre parenthèses)

Dans cette étude les sujets n'ont pas diminué ou augmenté leurs revenus criminels lorsqu'ils sont engagés dans une cohabitation matrimoniale. La seule autre étude comparable aboutit à des résultats contraires (Uggen et Thompson, 2003). Mais il est possible que ces résultats divergents soient imputables à des différences des échantillons : $55 \%$ des sujets de Uggen et Thompson étaient des toxicomanes et des décrocheurs scolaires et les délinquants sélectionnés avaient été seulement condamnés à une peine de probation. Nos résultats sont compatibles avec ceux de Horney et coll. (1995) qui montrent que la relation «maritale » (« live with wife ») a des effets régulateurs sur certains types de délits (les agressions), mais pas d'effets significatifs sur la délinquance de trafic ou les crimes contre la propriété (c'est-à-dire les plus susceptibles de procurer des revenus); en revanche la relation avec une « conjointe » (« live with girlfriend ») encourage la délinquance de trafic. L'étude longitudinale de Kazemian et Le Blanc (2007) indiquent en outre que les relations maritales ne semblent pas influencer les risques effectifs d'arrestation, une composante complémentaire de leur performance criminelle.

Quelque soit leur penchant («manque de contrôle de soi »), leur âge ou encore de la taille de leur réseau criminel, les délinquants augmentent leurs revenus criminels lorsque leurs revenus légitimes chutent et vice versa $(B=-0,04 ; \mathrm{p} \leq, 01)$. On peut supposer que les mois où les revenus augmentent, les délinquants passent plus de temps au travail (moins de temps pour le crime). Comme ils disposent d'une plus grande marge de manœuvre financière, ils peuvent aussi ou bien modérer la fréquence de leurs délits ou bien sélectionner les délits les moins risqués.

Le modèle 1 du Tableau 3 souligne l'impact des mesures de surveillance sur les revenus criminels. Il est plus probable que les gains illicites chutes durant les mois où les délinquants sont en libération conditionnelle, en probation ou en maison de transition $(B=-0,49 ; p \leq, 01)$. Cet effet est appréciable. Les épisodes de surveillance diminuent de $31 \%$ les gains criminels mensuels. C'est donc dire qu'un délinquant qui génère en moyenne $2000 \$$ de revenu criminel avant d'être sous surveillance verra ses gains mensuels chuter à $646 \$$ les mois où il est sous surveillance ${ }^{3}$. Dans ces circonstances, les risques d'être arrêté ou encore de retourner en prison sont accentués. Il n'est donc pas surprenant de constater que les délinquants modèrent leurs activités criminelles dans de telles situations. Cet effet «modérateur » de la surveillance formelle est observable dans l'étude de McGloin et ses collègues (2007), qui montrent que les délinquants ont moins tendance à diversifier leurs activités criminelles durant les mois de surveillance.

Par ailleurs, les gains criminels diminuent lorsque les délinquants ont moins de succès à éviter les appréhensions policières et augmentent lorsqu'ils ont plus de succès $(\mathrm{B}=0,98 ; \mathrm{p} \leq, 001)$. Contrairement à ce que propose l'étude de Kazemian et Le Blanc (2007), nous trouvons un lien entre l'habilité à éviter les coûts du crime et les revenus criminels mensuels. Les délinquants qui réussissent à ne pas se faire prendre pour leurs crimes ne se contentent pas d'une seule victoire. La confiance qu'ils acquièrent par les expériences d'impunité les incite à prendre des moyens pour augmenter leurs revenus criminels. À l'opposé, les échecs à éviter les sanctions, tout comme

\footnotetext{
${ }^{3} 3,3 \log (2000 \$)-0,49=2,99 \log (645,65 \$)$
} 
les épisodes de surveillance pénale, montrent l'effet dissuasif des mesures d'intervention formelles. Il reste à savoir si ces facteurs prédisent également les épisodes d'inactivité criminelle.

Les délinquants qui bénéficient d'un réseau criminel plus important retirent des gains criminels plus élevés que ceux qui ne possèdent pas un tel capital social $(B=0,03, p \leq, 05)$. Lorsque l'on tient constante la diversification mensuelle des activités criminelles, cet effet disparaît, tout comme celui des revenus légitimes. Les gains criminels varient en fonction de la diversification criminelle $(\mathrm{B}=0,86 ; \mathrm{p} \leq, 001)$. Les mois où le nombre d'activités illégales dans lesquelles les contrevenants sont impliqués augmente se traduisent par des gains criminels supérieurs. À l'inverse, lorsque le nombre d'activités criminelles dans lesquelles s'investissent les contrevenants se restreint, pour quelconques raisons, les revenus du crime chutent. En somme, un réseau criminel plus étendu augmente les opportunités criminelles (Hochstetler, 2002; Morselli et Tremblay, 2004; Tremblay, 1993) et les mois où les délinquants diversifient leurs activités se traduisent par des revenus mensuels plus élevés.

La consommation quotidienne d'alcool ne contraint pas la performance criminelle. Celle-ci tend plutôt à être un indicateur du style de vie et de leurs revenus : les délinquants qui réussissent mieux ont davantage d'argent à dépenser, et il semble donc plus enclin à faire la fête et à se livrer à une consommation d'alcool plus régulière dans les bars et ailleurs. Force est d'admettre qu'à court terme le succès criminel s'explique par le contexte ou encore les causes situationnelles. Les modèles sur la performance criminelle montrent l'importance des circonstances, notamment celles de la vie déviante. En tenant constantes ces circonstances, les indicateurs du contrôle social ont peu de pouvoir prédictif. Il reste à voir si ces résultats se transposent dans les modèles des épisodes d'inactivités.

\section{Les épisodes d'inactivité criminelle}

Les résultats précédents indiquent que les délinquants diminuent leurs revenus lorsqu'ils sont assujettis à une supervision pénale et lorsque leur succès à éviter les appréhensions policières diminue. Jusqu'à quel point ces circonstances influencent-elles aussi l'inactivité dans le crime? Pour évaluer cette influence, on doit incorporer dans l'analyse leurs épisodes d'inactivité. Nos modèles présentés au Tableau $4^{4}$ incorporent les épisodes d'inactivité et cherchent à expliquer les mois où les contrevenants s'abstiennent volontairement de commettre des crimes.

Tableau 4 : Les circonstances de vie et les caractéristiques des participants sur les épisodes d'inactivité criminelle : modèle linéaire hiérarchique généralisé (MLHG), coefficients logistiques $(\gamma)$ et Odds Ratio.

\begin{tabular}{|c|c|c|c|c|}
\hline \multirow[t]{2}{*}{ INACTIVITÉ CRIMINELLE } & \multicolumn{2}{|c|}{$\frac{\text { MODËLE 4 }}{\text { Contrôle sociaux }}$} & \multicolumn{2}{|c|}{$\frac{\text { MODĖLE 5 }}{\text { Implication criminelles }}$} \\
\hline & $\gamma$ & Odds Ratio & $\gamma$ & Odds Ratio \\
\hline \multicolumn{5}{|l|}{ NIVEAU $1(\mathrm{~N}=5125)$} \\
\hline Cohabitation conjugale & $\begin{array}{l}-0,09 \\
(0,15)\end{array}$ & 0,862 & $\begin{array}{l}-0,11 \\
(0,46)\end{array}$ & 0,897 \\
\hline Revenu légitime & $\begin{array}{l}0,22 * * * \\
(0,08)\end{array}$ & 1,250 & $\begin{array}{l}0,24 * * * \\
(0,07)\end{array}$ & 1,266 \\
\hline
\end{tabular}

${ }^{4}$ À défaut des modèles linéaires hiérarchiques, l'utilisation des modèles linéaires hiérarchiques généralisés ne renseigne pas sur la variance expliquée de chacun des niveaux, l'hétéroscédasticité des termes d'erreur au premier niveau en est la cause. 
Surveillance pénale

NIVEAU $2(\mathbf{N}=172)$

Âge

Niveau de scolarité

Précocité de la carrière criminelle

Manque de contrôle de soi

Consommation quotidienne d'alcool

Utilisation de drogues dures

Réseau criminel

Revenus criminels moyens

Succès moyen à éviter les contacts policiers

Diversification criminelle moyenne

\begin{tabular}{ll|ll}
0,15 & 0,862 & 0,80 & 2,223 \\
$(0,18)$ & & $(0,48)$ & \\
& & & \\
0,01 & 1,010 & 0,01 & 1,014 \\
$(0,03)$ & & $(0,03)$ & \\
$-0,12$ & 0,921 & 0,29 & 1,340 \\
$(0,54)$ & & $(0,52)$ & \\
0,00 & 1,005 & 0,00 & 1,005 \\
$(0,03)$ & & $(0,03)$ & \\
$-0,02$ & 0,981 & $-0,01$ & 0,998 \\
$(0,02)$ & & $(0,02)$ & \\
$-0,54$ & 0,545 & $-0,40$ & 0,668 \\
$(0,47)$ & & $(0,45)$ & \\
$-0,14$ & 0,904 & $-0,16$ & 0,846 \\
$(0,47)$ & & $(0,43)$ & \\
$-0,03 *$ & 0,964 & $-0,02$ & 0,983 \\
$(0,02)$ & & $(0,02)$ & \\
& & $-0,21 * *$ & 0,815 \\
& & $(0,07)$ & \\
& & $-1,37 * *$ & 0,931 \\
& & $(0,88)$ & \\
& & $-0,94 * *$ & 0,391 \\
& $(0,28)$ & \\
\end{tabular}

$*=\overline{\mathrm{p}<, 05 ; * *=\mathrm{p}<, 01 ; * * *=\mathrm{p}<, 001 \text { (les erreurs types sont entre parenthèses) }}$

Les résultats des analyses résumées dans le Tableau 4 indiquent que les délinquants sont plus enclins à cesser leurs activités criminelles durant les mois ou leurs revenus légitimes augmentent (Odds Ratio $=1,250 ; \mathrm{p} \leq, 01$ ). Ce résultat corrobore les travaux de (Horney et coll.1995; Griffin et Armstrong, 2003, Uggen et Thompson, 2003; McGloin et coll., 2007). En revanche, les mois de cohabitation conjugale n'ont pas plus d'effet sur la probabilité qu'ils cessent temporairement leurs activités criminelles qu'ils n'en avaient sur les fluctuations de leurs revenus criminels. Leur capital social criminel, c'est-à-dire le nombre de leurs partenaires en délinquance, diminue la fréquence de leurs épisodes d'inactivité (Odds Ratio $=0,969 ; \mathrm{p} \leq, 05$ ). Un délinquant qui ne possède pas de contacts dans les milieux criminels à 10,3 fois plus de chances d'être inactif durant la période d'observation qu'un individu qui en compte dix

On a observé que le capital social criminel permettait aux délinquants d'élargir de répertoire de leurs opportunités criminelles, c'est-à-dire de se diversifier. On pourrait penser que cette diversification diminuerait la fréquence de leurs épisodes d'inactivité. Pour vérifier les mérites de cette proposition, nous avons crée trois nouvelles variables statiques qui caractérisent à la fois le degré de performance criminelle (revenus illicites et expérience d'impunité) et la diversification des activités durant la période fenêtre (modèle 5). Les nouvelles mesures créées à partir de nos indicateurs dynamiques du Tableau 3 représentent la moyenne de ces paramètres durant les mois où les délinquants étaient actifs. Ces indicateurs permettent d'examiner jusqu'à quel point les revenus criminels moyens, le succès moyen à éviter les contacts policiers et la diversification criminelle moyenne influencent la décision des délinquants d'interrompre leurs activités 
criminelles. Les résultats de cette analyse montrent que la fréquence des épisodes d'inactivité varie en fonction inverse de la capacité des délinquants de diversifier les activités criminelles dans lesquelles ils s'investissent (Odds Ratio $=0,391 ; p \leq, 01$ ), des revenus criminels moyens qu'ils retirent (Odds Ratio $=0,815 ; \mathrm{p} \leq, 01)$ et de leur aptitude à éviter d'être arrêté (Odds Ratio $=0,931 ; p \leq, 01$ ). À l'inverse, les délinquants dont les trajectoires sont truffées d'échecs (revenus médiocres, appréhension, arrestation et incarcération) et qui bénéficient d'un nombre limité d'opportunités criminelles sont plus enclins à connaître une trajectoire de délinquance intermittente. Autrement dit, les délinquants ajustent la fréquence de leurs épisodes d'inactivité au succès passé ou escompté des gains que leurs délits leur procurent (Hochstetler, 2002).

\section{Les caractéristiques des délinquants}

Horney et coll. (1995) rapportent que les caractéristiques individuelles des délinquants (âge, niveau d'instruction) n'ont pas d'effet direct à court terme sur la fréquence des délits commis. Uggen et Thompson (2003) n'en tiennent même pas compte. Ils justifient ce choix par leur homogénéité de ces caractéristiques au sein des populations délinquantes. Toutefois, McGloin et ses collègues (2007) ont montré que certaines caractéristiques comme la précocité de la trajectoire de délinquance avaient un impact. Cette étude montre que la taille du réseau criminel des délinquants a un effet crucial sur les fluctuations de leurs revenus criminels et sur la fréquence de leurs épisodes d'inactivité. L'effet de la taille du réseau disparaît toutefois lorsque l'on tient constant les variations mensuelles de la diversification criminelle (modèle 3 et 5).

L'accès à une diversité d'opportunités criminelles dépend donc de la taille du réseau criminel. Ce résultat nous a incités à analyser de manière plus systématique les liens entre les caractéristiques individuelles des délinquants et la dynamique des changements qui se produisent au cours de leur trajectoire de délinquance (Tableau 5).

Cette analyse des effets dits indirects est instructive. L'hypothèse générale est que ces changements ne sont pas des variations « aléatoires » : certaines caractéristiques dites «statiques » permettent aux délinquants de s'adapter plus facilement ou moins facilement aux changements de circonstances ou aux perturbations qui affectent leur trajectoire de délinquance. Plusieurs travaux soulignent par ailleurs l'importance de la précocité de la délinquance dans la prédiction de la trajectoire criminelle à l'âge adulte; l'âge au premier crime prédit notamment la persistance et la fréquence des activités criminelles (Le Blanc et Fréchette, 1989; Loeber et Le Blanc, 1990). Le Tableau 5 révèle que les délinquants qui débutent tôt leur carrière sont plus à risque de se trouver sous surveillance pénale. Comme les revenus des délinquants sous surveillance tendent à diminuer, les délinquants qui ont débuté leurs activités criminelles précocement sont plus susceptibles d'afficher des décroissances dans leurs revenus criminels mensuels que ceux qui se sont lancés plus tardivement. À noter que les mois de cohabitation maritale sont moins fréquents pour les délinquants qui débutent leur trajectoire de délinquance à un âge précoce que pour ceux qui débutent à un âge plus tardif. Il est difficile d'expliquer ce résultat. Une possibilité est qu'ils ont « moins » à offrir à leurs partenaires. Une autre possibilité est que leurs relations de cohabitation maritale sont moins durables. Ou encore que leurs séjours en prison durant la période d'observation (ou avant) sont plus fréquents ${ }^{5}$ et que ces « retraits » nuisent à la durée et/ou à la fréquence de leurs relations de cohabitation maritale. Nos analyses

\footnotetext{
${ }^{5}$ La corrélation entre les mois d'incarcération durant la période d'observation et la précocité est de, 211 ( $\left.\mathrm{p}=, 005\right)$
} 
n'ont pas pour fonction de trancher entre ces scénarios, mais de suggérer des pistes intéressantes de recherche.

Tableau 5 : Interrelations entre les circonstances de vie et les caractéristiques des délinquants : coefficients de régression non standardisés (b)

\begin{tabular}{rccccc}
\hline Âge & $\begin{array}{c}\text { Cohabit. } \\
\text { conjugale }\end{array}$ & $\begin{array}{c}\text { Revenu } \\
\text { légitime }\end{array}$ & $\begin{array}{c}\text { Surveillance } \\
\text { pénale }\end{array}$ & $\begin{array}{c}\text { Succès à éviter } \\
\text { contacts pol. }\end{array}$ & Diversification \\
Niveau de scolarité & $\mathbf{X}$ & $\mathbf{X}$ & $\mathbf{X}$ & $0,01 *(0,00)$ & $\mathbf{X}$ \\
$\begin{array}{r}\text { Précocité de la } \\
\text { carrière criminelle }\end{array}$ & $0,09 *(0,04)$ & $\mathbf{X}$ & $-0,11 *(0,05)$ & $\mathbf{X}$ & $\mathbf{X}$ \\
$\begin{array}{r}\text { Manque de contrôle } \\
\text { de soi }\end{array}$ & $\mathbf{X}$ & $\mathbf{X}$ & $\mathbf{X}$ & $\mathbf{X}$ & $0,01 *(0,00)$ \\
$\begin{array}{r}\text { Consommation } \\
\text { quotidienne d'alcool }\end{array}$ & $\mathbf{X}$ & $\mathbf{X}$ & $\mathbf{X}$ & $\mathbf{X}$ & $\mathbf{X}$ \\
$\begin{array}{r}\text { Utilisation de drogues } \\
\text { dures }\end{array}$ & $\mathbf{X}$ & $\mathbf{X}$ & $\mathbf{X}$ & $\mathbf{X}$ & $\mathbf{X}$ \\
Réseau criminel & $0,04 *(0,02)$ & $\mathbf{X}$ & $\mathbf{X}$ & $\mathbf{X}$ & $0,01 * *(0,00)$ \\
\hline$*, 05 ; * *=\mathrm{p}<, 01 ; * * * *$ \\
\hline
\end{tabular}

Le Tableau 5 montre aussi que les délinquants plus âgés et plus instruits ont davantage de succès à éviter les contacts policiers. Puisque le succès à éviter les contacts policiers a un impact direct sur les revenus criminels mensuels et les épisodes d'inactivité criminelle, il s'ensuit que, toutes choses étant égales par ailleurs, les délinquants plus âgés et plus instruits connaitraient des variations ascendantes de leurs revenus criminels mensuels lorsqu'ils sont actifs et qu'ils seraient également moins enclins à cesser leurs activités criminelles.

On ne s'étonnera pas que la fréquence de consommation d'alcool n'ait pas d'impact indirect sur la manière dont les délinquants s'adaptent aux changements de circonstances qui affectent leur trajectoire de délinquance puisque nos analyses antérieures indiquaient que cette consommation était davantage causée par les revenus criminels que l'inverse - une dépense ostentatoire de leur degré de réussite relative à retirer des gains de leurs activités criminelles («j’ai de l'argent, donc je le bois »), un résultat conforme à la théorie de la «vie festive » proposée par Cusson (2005).

L'hypothèse selon laquelle l'impulsivité, le présentisme et le goût du risque pourrait expliquer la volatilité ou l'instabilité des épisodes de réussite dans une trajectoire de délinquance doit être rejetée. Il est possible que la volatilité des revenus criminels soit intrinsèque à la vie criminelle et une conséquence inévitable de leur illégalité et que les caractéristiques individuelles ne puissent pas minimiser cet aléa (voir Morselli, 2009, p.11).

\section{CONCLUSION}

Les recherches sur les trajectoires de délinquance qui adoptent une perceptive dynamique et qui exploitent la stratégie des calendriers sont peu nombreuses. Elles sont cependant nécessaires pour plusieurs raisons. Elles devraient être intégrées aux études prospectives longitudinales. Même lorsqu'elles sont rétrospectives, elles permettent de comprendre comment les délinquants 
s'adaptent aux circonstances changeantes de leur vie ou de l'impact de ces circonstances sur le degré de leur motivation à persévérer dans leurs activités criminelles. La séquence des crimes qu'ils commettent n'est pas constituée d'évènements isolés les uns des autres ou de coïncidences fortuites, mais d'épisodes interreliés de choix successifs qui poursuivent les mêmes finalités (Hochstetler, 2002). Dans cette étude, la finalité retenue est celle de leur réussite relative à retirer des gains tangibles de leurs activités illicites. Mais on pourrait généraliser l'approche en envisageant d'autres types de finalité (par exemple le statut ou le prestige dans un groupe ou une organisation criminelle).

Une notion centrale de cette étude est celle des épisodes d'inactivité délinquante. La thèse selon laquelle la délinquance serait une activité continue est généralement erronée (la plupart des délinquants ont une trajectoire délinquante intermittente, alternant des épisodes d'activité et d'inactivité). Cette intermittence est caractéristique non seulement des délinquants « occasionnels », mais aussi des délinquants «chroniques ». Le modèle de trajectoire de délinquance proposé par Blumstein et coll. (1986) a l'avantage de nous inciter à croiser l'analyse des paramètres de cette trajectoire (précocité, co-délinquance, diversité délictuelle, durée, gravité, cadence individuelle des délits commis, diversité délictuelle). Le premier inconvénient de ce modèle est qu'il ignore les pressions régulatrices qui s'exercent sur les délinquants changent au fil du temps (emploi, mariage, service militaire, instruction ou « retours à l'école) peuvent avoir un impact décisif («turning points ») sur la durée d'une trajectoire de délinquance (Sampson et Laub, 1993). Dans cette étude nous nous sommes attardés aux trois premiers paramètres, mais avons doté les délinquants d'une « finalité » (l'aptitude à retirer des gains tangibles de leurs délits et l'aptitude à éviter les arrestations). Nous avons pris en considération à la fois les changements de vie qui sont susceptibles d'inciter les délinquants à retourner dans le « droit chemin » (relation maritale, emploi légitime, surveillance pénale) que les circonstances susceptibles d'encourager la pérennité des activités criminelles (expérience d'impunité, opportunité criminelle).

Les modèles de trajectoire de délinquance proposés par Blumstein et ses collègues ou par Sampson et Laub partagent une dernière lacune : ils postulent que la trajectoire de délinquance est continue ou que les épisodes d'activité délinquance sont aléatoires et se bornent simplement à signaler ces épisodes d'inactivité ne doivent pas être interprétés comme des signes avant-coureurs d'un désistement « définitif » (Piquero, 2004; Kazemian et Maruna, 2009). La caractéristique des analyses que propose cet article n'est pas simplement qu'elle est « dynamique » au sens où les facteurs qui influencent le comportement des délinquants «varient » au cours de leur trajectoire. Sa caractéristique centrale est qu'elle est hiérarchique c'est-à-dire qu'elle compare les délinquants à eux-mêmes.

À l'instar de Uggen et Thompson (2003), nos résultats montent que les épisodes d'emplois légitimes expliquent non seulement les variations dans l'intensité de l'activité criminelle (les revenus illégitimes), mais aussi la motivation des délinquants à cesser leurs activités criminelles. Cette cessation n'est sans doute pas « définitive ». Dans cet échantillon de détenus fédéraux, $69 \%$ ont trouvé un emploi durant au moins un des 36 mois qui ont précédé leur incarcération actuelle. Nos analyses n'ont pas pour fonction de montrer que les délinquants qui occupent un emploi légitime (oui ou non) durant la période étudiée sont moins enclins à commettre des délits ou moins incités à majorer leurs revenus criminels (indicateur statique); elles disent plutôt que parmi ceux qui ont décidé de prendre un emploi durant la période d'observation, les mois durant lesquels ils travaillent sont aussi les mois durant lesquels leurs revenus criminels diminuent 
(indicateur dynamique). De manière analogue on peut comparer les délinquants sur la nature plus ou moins intermittente de leurs trajectoires de délinquance. Dans cet échantillon de détenus fédéraux, $38 \%$ n’ont jamais été « inactifs ». Mais pour la majorité de ceux qui ont été inactifs pendant au moins un des 36 mois qui ont précédé leur incarcération actuelle, ils avaient davantage de chances d'être inactifs durant les mois où ils travaillent que durant les mois où ils ne travaillaient pas. L'analyse hiérarchique tient constantes les différences entre délinquants et compare leur comportement durant un moment de leur trajectoire à leur propre comportement durant la période qui précède ou qui suit ce moment. Le moment étant défini ici comme un mois durant laquelle une circonstance est présente ou absente, par exemple, mois de cohabitation maritale, mois où le sujet travaille où le sujet commet des délits). La difficulté propre à une analyse «multi-niveaux » ou « hiérarchique » est de ne pas basculer, subrepticement, d'une interprétation qui compare les individus entre eux à une interprétation qui, tenant constantes ces différences, compare les individus à eux-mêmes. Nos résultats indiquent aussi que les délinquants sont davantage enclins à être «inactifs » durant les mois où ils sont assujettis à une surveillance pénale (libération conditionnelle, probation ou maison de transition) et davantage enclins à diminuer leurs revenus criminels.

Ces résultats impliquent que les programmes visant à favoriser l'employabilité des ex-détenus ou la surveillance pénale des agents de réinsertion sociale procurent des bénéfices tangibles. Ces bénéfices sont peut-être temporaires, mais un bénéfice temporaire est plus encourageant que son absence. Les mois d'inactivité ne sont pas garants d'une réhabilitation définitive, mais là encore une réhabilitation temporaire est préférable à son absence et on peut penser que des séquences répétées de réhabilitation temporaire peuvent inciter les agents de réinsertion sociale à se fixer comme objectif d'étape de prolonger ces séquences.

Cette étude n'explore pas l'impact spécifique d'une perte d'emploi, l'attachement ou l'investissement personnel qu'il représente pour le délinquant, pas plus qu'elle n'examine l'impact d'un changement de conjointe ou d'une séparation, la naissance d'un enfant ou encore le décès d'une personne proche. Plusieurs cambrioleurs rencontrés dans l'étude d'Hochstetler (2002) associent les événements pénibles qui sont survenus dans leur vie, comme la perte de leur emploi, des dettes légales, le divorce et l'expulsion d'un logement, à leur décision de passer à l'acte. Cet article n'explore pas non plus la combinaison de tels évènements (divorce durant l'incarcération par exemple). Étendre le registre des évènements susceptibles d'affecter une trajectoire de délinquance permettrait de jauger la robustesse et les mérites de l'approche que nous avons adoptée dans cet article et de nuancer sans doute certains de ses résultats. Notre étude suggère que parmi ceux qui entretenaient des relations de cohabitation maritale, les mois de cohabitation maritale n'étaient pas nécessairement les mois où ils étaient enclins à cesser leurs activités délinquantes ou, s'ils étaient actifs, à modérer leurs revenus criminels. Mais l'enquête qu'utilise cette étude n'a pas incorporé l'intensité de la relation « amoureuse » ou sa qualité dans le registre des données de calendrier (Sampson, Laub et Wimer, 2006). L'approche que nous proposons pourrait se révéler appropriée dans le cadre de recherches qui ne s'intéressent pas particulièrement au degré de performance utilitaire des délinquants (les gains matériels qu'ils procurent). Les études qui évaluent le degré de dangerosité des délinquants et les outils actuariels couramment utilisés se sont peu intéressées à analyser l'impact des circonstances immédiates qui caractérisent les épisodes de récidive ou de « passage à l'acte » (Lussier et Davies, 2011; Ouellet, 2012). Si on en juge par les résultats de cette étude, ils gagneraient à le faire et à adopter une stratégie d'analyse appropriée à inventorier cet impact. 
Une limite de l'approche hiérarchique est qu'elle requiert un niveau minimum de la fréquence de base pour considérer les circonstances de vie dans les modélisations. Les décès, divorces ou tentatives de suicide sont des évènements trop rares dans la majorité des trajectoires de vie (surtout les trajectoires relativement courtes qui s'étalent sur quelques dizaines de mois) pour se prêter à une telle analyse. Mais on peut y remédier indirectement en incorporant une mesure répétée d'un état émotionnel ( humeurs dépressives ») ou d'une relation émotionnelle (fréquente relative des disputes avec la conjointe). Une autre limite de cette étude est son incapacité à capter la durée des effets identifiés. La durée de l'effet dissuasif qui accompagne la sortie de prison s'estompe-t-elle après un certain temps?

L'examen détaillé des épisodes d'activité et d'inactivité criminelles nous apprend que les décisions des délinquants sont fortement influencées par les expériences présentes (les circonstances de la vie) et passées (leur succès ou leur échec à éviter les appréhensions policières). Les délinquants persistants apprennent de leurs expériences et utilisent les connaissances acquises pour améliorer leur performance et pour réduire les risques liés à leurs activités. Dans cette étude, les délinquants qui sont habiles à se faire des contacts, à éviter les appréhensions policières et qui retirent des gains appréciables de leurs activités criminelles sont moins enclins à interrompre temporairement leurs activités criminelles. En outre, l'expérience et l'instruction sont des outils qui permettent d'éviter plus facilement les appréhensions policières. Il en va de même pour la diversification criminelle, qui dépend de l'étendue du réseau des criminels. La performance et la participation aux crimes lucratifs semblent être influencées davantage par les compétences et les habiletés, ce qui concorde par ailleurs avec la position de plusieurs auteurs (Becker, 1962; McCarthy et Hagan, 2001; Morselli et Tremblay, 2004; Tremblay et McCarthy, 2006).

L'étude d'une trajectoire de délinquance bénéficie d'une intégration des perspectives de la trajectoire de vie (Sampson et Laub, 1993) et des perspectives développementales (sur cette intégration, voir Farrington, 2005). L'étude suggère que l'analyse de calendriers est une stratégie précise pour opérer cette intégration. Elle utilise les revenus criminels mensuels de délinquants qui ont commis au moins un délit potentiellement lucratif pendant au moins trois mois (pas nécessairement consécutifs) pour opérer cette intégration. Le critère d'éligibilité n'est pas particulièrement restrictif. Les résultats de cette intégration sont instructifs. Nos premières analyses (Tableaux 3 et 4 ) suggéraient que la taille du réseau de délinquants qui gravitent autour de nos sujets ou la précocité de leur trajectoire de délinquance n'avaient pas d'impact sur les fluctuations de leurs revenus criminels sur la fréquence relative de leurs épisodes d'activité délinquante. Morselli et coll. (2006) montrent que la rencontre d'un mentor a un impact sur le capital social criminel des délinquants (la taille de leurs réseaux de partenaires potentiels en délinquance) et celui-ci a, à son tour, un impact positif sur la diversité du répertoire de leurs revenus criminels et les revenus qu'ils en retirent. L'enquête sur laquelle se base cette étude ne permet pas de mesurer les fluctuations mensuelles de ce capital social. Mais l'impact durable de l'effet de ces mentors justifie que l'on puisse considérer ce capital social comme une constante durant la période relativement courte (36 mois) de la trajectoire délinquante observée. Les résultats de cet article indiquent que les délinquants qui ont davantage de capital social criminel ont des revenus criminels mensuels moyens plus élevés tout au long de cette trajectoire et que leurs expériences mensuelles d'impunité sont également plus élevées. Mais nos analyses subséquentes indiquent que les délinquants qui débutent tôt leur carrière sont plus souvent sous 
surveillance pénale que ceux qui débutent plus tardivement. Comme les revenus des délinquants sous surveillance tendent à diminuer, les fluctuations des revenus criminels mensuels de ces délinquants déclineront plus fréquemment que celle des délinquants moins précoces. Elles indiquent aussi que les délinquants plus âgés et plus instruits ont davantage de mois d'activité où leurs expériences d'impunité sont fréquentes. Lorsque le sentiment d'impunité augmente, les délinquants seront incités à majorer leurs revenus criminels, de sorte que les délinquants plus âgés et plus instruits auront davantage de chances de connaître des hausses de leurs revenus mensuels que ceux qui n'ont pas ces caractéristiques. Ces analyses combinent trois registres ou perspectives. La perspective à long terme de la trajectoire de vie (le capital social que les délinquants ont cumulé depuis leur début dans le crime); la perspective à court terme des circonstances changeantes durant cette trajectoire, et la perspective développementale (précocité de la délinquance, âge et manque de contrôle de soi).

\section{RÉFÉRENCES}

Patricia A. Adler and Peter Adler

1983 Shifts and oscillations in deviant careers: the case of upper-level drug dealers and smugglers. Social Problems 31: 195-207.

Ahn, Chul W., Alfred Blumstein and Mark Schervish 1990 Estimation of Arrest Careers Using Hierarchical Stochastic Models. Journal of Quantitative Criminology 6: 131-152.

Becker, Gary S.

1962 Investment in Human Capital-A Theoretical Analysis. Journal of Political Economy 70: $9-49$.

Bouchard, Martin and Frédéric Ouellet

2011 Is small beautiful? The link between risks and size in illegal drug markets. Global Crime 12, 1: 70-86.

Bradburn, Norman M., Lance J. Rips and Steven K. Shevell 1987 Answering Autobiographical Questions: The Impact of Memory and Inference on Surveys. Science 236: 157-161.

Chaiken, Marcia R. and Jan M. Chaiken

1985 Who gets caught doing crime? Los Angeles: Bureau of Justice Statistics (U.S.)

Charest, Mathieu

2004 Peut-on se fier aux délinquants pour estimer leurs gains criminels. Criminologie 37 : 63 88.

Charest, Mathieu and Pierre Tremblay

2009 Immobilité sociale et trajectoires de délinquance. Revue française de sociologie 50(4), 693-718. 
Cusson, Maurice

2005 La délinquance, une vie choisie. Montréal : Hurtubise.

Dupéré, Véronique, Éric Lacourse, Frank Vitaro and Richard E. Tremblay

2007 Méthodes d'analyse du changement fondées sur la trajectoire de développement individuelle : modèles de régression mixtes paramétrique et non paramétrique. Bulletin de Méthodologie Sociologique 97: 1-28.

Farrington, David P.

2005 The integrated cognitive antisocial potential (ICAP) theory. In: David P. Farrington (ed), Integrated developmental and life-course theories of offending. New Brunswick, NJ:

Transaction.

Farrington, David P. and Donald J. West

1995 Effects of marriage, separation and children on offending by adult males. In: J. Hagan

(ed), Current perspectives on aging and the life cycle. Volume 4: Delinquency and disrepute in the life course. Greenwich: JAI.

Fisher, Ronald P. and Edward R. Geiselman

1989 Field Test of the Cognitive Interview: Enhancing the Recollection of Actual Victims and Witnesses of Crime. Journal of Applied Psychology 74: 722-727.

Freedman, Deborah, Arland Thornton, Donald Camburn, Duane Alwin and Linda YoungDeMarco

1988 The Life History Calendar: A Technique for Collecting Retrospecitve Data. Sociological Methodology 18: 37-68.

Gottfredson, Michael R.

2005 Offender classifications and treatment effects in developmental criminology: A

propensity/event consideration. Annals of the American Academy of Political and Social Science 602: 46-56.

Gottfredson, Michael R. and Travis Hirschi

1990 A General Theory of Crime. Palo Alto: Stanford University Press.

Griffin, Marie L. and Gaylene S. Armstrong

2003 The Effect of Local Life Circumstances on Female Probationer's Offending. Justice

Quarterly 20: 213-239.

Grasmick, Harold G., Charles R. Tittle, Robert J. Bursik, Jr. and Bruce J. Arneklev

1993 Testing the Core Empirical Implications of Gottfredson and Hirschi's General Theory of Crime. Journal of Research in Crime and Delinquency 30: 5-29.

Hagan, John and Bill McCarthy

1997 Mean Streets: Youth Crime and Homelessness. Cambridge: Cambridge University Press.

Hirschi, Travis and Micheal R. Gottfredson 
1983 Age and the explanation of crime. American Journal of Sociology 89: 552-584.

Hochstetler, Andy

2002 Sprees and runs: The construction of opportunity in criminal episodes. Deviant Behavior 23:45-74.

Horney, Julie and Ineke H. Marshall

1991 Measuring Lambda through Self-Reports. Criminology 31: 471-495.

Horney Julie, D. Wayne Osgood and Ineke H. Marshall

1995 Criminal Careers in the Short-Term: Intra-Individual Variability in Crime and its Relation to Local Life Circumstances. American Sociological Review 60: 655-673.

Jacob, Bruce A.

1996 Crack dealers' apprehension avoidance techniques: A case of restrictive deterrence.

Justice Quarterly 13: 359-38.

Johnson, Bruce D., Mangai Natarajan and Harry Sanabria

1993 Successful criminal careers: toward an ethnography within the rational choice perspective. In: Ronald V. Clarke and Marcus Felson (eds.) Routine Activity and Rational Choice. New Brunswick: Transaction Publishers.

Kazemian, Lila and Marc Le Blanc

2007 Differential Cost Avoidance and Successful Criminal Careers: Random or Rational?

Crime and Delinquency 53: 38-63.

Kazemian, Lila and Shadd Maruna

2009 Desistance from crime. In: Marvin D. Krohn, Gina Penly Hall, Alan J. Lizotte (eds.)

Handbook on crime and deviance. New York: Springer.

Laub, John H. and Robert J. Sampson

2003 Shared Beginnings, Divergent Lives: Delinquent Boys to Age 70. Cambridge: Harvard University Press.

Le Blanc, Marc and Marcel Fréchette

1989 Male criminal activity from childhood through youth: Multilevel and developmental perspectives. New York: Springer-Verlag.

Levitt, Steven D. and Sudhir A. Venkatesh

2000 An economic analysis of a drug-selling gang's finances. The Quarterly Journal of

Economics, 115 (3), 755-789.

Loeber, Rolf and Marc Le Blanc

1990 Toward a developmental criminology. In: Norval Morris and Micheal Tonry (eds). Crime and justice. Chicago: University of Chicago Press.

Luke, Douglas A. 
Lussier, Patrick and Garth Davies

2011 A person-oriented perspective on sexual offenders, offending trajectories, and risk of recidivism: A new challenge for policymakers, risk assessors, and actuarial prediction?

Psychology, Public Policy, and Law, 17 (4), 530-561.

McCarthy, Bill and John Hagan

2001 When crime pays: Capital, competence, and criminal success. Social Forces 79 : 10351059 .

McGloin, Jean M., Christopher J. Sullivan, Alex R. Piquero and Travis C. Pratt 2007 Explaining Qualitative Change in Offending: Revisiting Specialization in the Short-Term. Journal of Research in Crime and Delinquency 44: 321-346.

MacKenzie, Doris L. and Spencer D. Li

2002 The impact of formal and informal control on criminal careers of probationers. Journal of Research in Crime and Delinquency 39: 243-276.

Matza, David

1967 Delinquency and drift. New York: John Wiley \& Sons, Inc.

Morselli, Carlo

2009 Inside criminal networks. New York: Springer.

Morselli, Carlo and Pierre Tremblay

2004 Délinquance, performance et capital social. Criminologie 42 : 773-804.

Morselli, Carlo and Pierre Tremblay

2010 Interviewing and Validity Issues in Self-Report Research with Incarcerated Offenders:

The Quebec Inmate Survey (2000-2001), pp. 68-83 In: Wim Bernasco (ed.) Offenders on

Offending: Learning About Crime From Criminals. New York: Willan.

Morselli, Carlo, Pierre Tremblay and Bill McCarthy

2006 Mentors and Criminal Achievement. Criminology 44: 17-43.

Nagin, Daniel S. and Kenneth C. Land

1993 Age, Criminal Careers, and Population Heterogeneity: Specification and Estimation of a

Nonparametric, Mixed Poisson Model. Criminology 31 : 327-362.

Ouellet, Frédéric

2012 La dynamique des séquences de rechute chez des joueurs excessifs et des joueurs délinquants. Criminologie, 45 (2), 51-85.

Peterson, Mark, and Harriet Braiker

1981 Who commits crime: a survey of prison inmates. Cambridge: Oelgeschlager, Gunn and Hain. 
Piquero, Alex R.

2004 Somewhere between persistence and desistance: The intermittency of criminal careers. In: Shadd Maruna et Russ Immarigeon (eds.). After crime and punishment: Pathways to offender reintegration (pp.102-125). New South Wales: Federation Press.

Piquero, Alex R.and Raymond Paternoster

1998 An application of Stafford and Warr's reconceptualization of deterrence to drinking and driving. Journal of Research in Crime and Delinquency, 35, 1: 3-39;

Piquero Alex R. and Greg Pogarsky

2002 Beyond Stafford and Warr's reconceptualization of deterrence: personal and vicarious experiences, impulsivity and offending behaviour. Journal of Research in Crime and

Delinquency, 39, 2: 153-186.

Piquero, Alex R. and Stephen G. Tibbetts

1996 Specifying Low Self-Control and Situational Factors in Offender Decision-Making.

Justice Quarterly 31: 601-631.

Pratt, Travis C. and Francis T. Cullen

2000 The Empirical Status of Gottfredson and Hirschi's General Theory of Crime: A MetaAnalysis. Criminology 38 : 931-964.

Robitaille, Clément

2004 À qui profite le crime ? Les facteurs de réussite délinquante. Criminologie 37: 233-262.

Sampson, Robert J. and John H. Laub

1990 Crime and Deviance over the Life Course: The Salient of Adult Social Bonds. American

Sociological Review 55: 609-627.

Sampson, Robert J. and John H. Laub

1993 Crime in the Making: Pathways and Turning Points throught Life. Cambridge: Harvard University Press.

Sampson, Robert J. and John H. Laub

2005 Developmental Criminology and Its Discontents: Trajectories of Crime from Childhood to Old Age. In: Robert J. Sampson and John H. Laub (eds). The Annals of the American Academy of Political and Social Science, Volume 602. Thousand Oaks: Sage Publications.

Sampson, Robert J., John H. Laub and Christopher WIMER

2006 Does Marriage Reduce Crime? A Counterfactual Approach to Within-Individual Causal Effects. Criminology 44: 465-508.

Shover Neal and Carol Y. Thompson

1992 Age, differential expectations, and crime desistance. Criminology 30: 89-104.

Stafford, Mark and Mark E. Warr 
1993 A reconceptualization of general and specific deterrence. Journal of Research in Crime and Delinquency 30: 123-135.

Thompson, Melissa and Christopher Uggen

2012 Dealers, Thieves, and the Common Determinants of Drug and Non-Drug Illegal Earnings. Criminology 50:1057 -87.

Tremblay, Pierre

1993 Searching for suitable co-offenders. In: Ronald V. Clarke and Marcus Felson (eds).

Routine activity and rational choice: advances in criminological theory. New Brunswick:

Transaction.

Tremblay, Pierre

2010 Le delinquent ideal : performance, discipline, solidarité. Montréal: Liber.

Tremblay, Pierre and Carlo Morselli

2000 Patterns in Criminal Achievement. Criminology 38: 633-660.

Uggen, Christopher and Melissa Thompson

2003 The socioeconomic Determinants of Ill-Gotten Gains: Within-Person Changes in Drug Use and Illegal Earnings. American Journal of Sociology 109: 146-185.

Warr, Mark E.

1998 Life-Course Transitions and desistance from crime. Criminology 36: 183-216.

Wilson, James Q. and Allan Abrahamse

1992 Does Crime Pay? Justice Quarterly 9: 359-377.

Willett, John B., Judith D. Singer and Nina C. Martin

1998 The design and analysis of longitudinal studies of development and psychopathology in context: Statistical models and methodological recommendations. Development and

Psychopathology 10: 395-426. 